\title{
A LARGE STRAIN EXPLICIT FORMULATION FOR COMPOSITES
}

\author{
JOSÉ B. RUBERT ${ }^{1}$, SERGIO OLLER ${ }^{2}$, ESTEVAM B. LAS CASAS ${ }^{3, *}$, \\ SERGIO P. B. PROENÇA ${ }^{1}$ AND EUGENIO OÑATE ${ }^{2}$ \\ ${ }^{1}$ Departamento de Engenharia de Estruturas, Escola de Engenharia de São Carlos da Universidade de São Paulo, \\ Av. Dr. Carlos Botelho, 1465, 13560-250 São Carlos SP, Brazil \\ ${ }^{2}$ International Center for Numerical Methods in Engineering (CIMNE), Universidad Politécnica de Cataluña, \\ Campus Norte UPC, 08034 Barcelona, Spain \\ ${ }^{3}$ Departamento de Engenharia de Estruturas, Escola de Engenharia da Universidade Federal de Minas Gerais, \\ Av. do Contorno 842, $2^{\circ}$ andar 30110-060 Belo Horizonte MG, Brazil
}

\begin{abstract}
SUMMARY
A geometrically non-linear formulation for composites and the resulting explicit dynamic finite element algorithm are presented. The proposed formulation assumes that small elastic and large plastic strains, being the anisotropy considered using tensors which map the model variables at each time step into an equivalent isotropic space, where the integration of the rate constitutive equations is performed. The evolution of the internal variables is calculated in the auxiliary spaces, taking into account the material non-linear behaviour, and the results mapped back to the real stress space. The updating of the mapping tensors for each new spatial configuration allows the treatment of general anisotropic materials under large strain and can be extended to treat multiphase composite materials using the mixing theory. The behaviour of the composite is dictated by the mechanical response of each substance, and the resultant model allows a fully non-linear analysis combining different material models, such as damage in one compounding substance, elastoplastic behaviour in the other, while a third substance behaves elastically. Copyright (C) 1999 John Wiley \& Sons, Ltd.
\end{abstract}

KEY WORDS: composites; anisotropy; mixing theory; constitutive model

\section{INTRODUCTION}

Composite structures strain and stress analysis normally involves the use of average material mechanical properties, or the study of each composite as a completely new material. The first approach is quite effective when all materials behave elastically, and the interaction between different phases is linearly dependent on their volumetric participation in the composite. In the second approach, material behaviour under loading is not obtained from the isolated properties

\footnotetext{
* Correspondence to: Estevam B. Las Casas, Departamento de Engenharia de Estruturas, Escola de Engenharia da Universidade Federal de Minas Gerais, Av. do Contorno 842, $2^{\circ}$ andar, 30110-060 Belo Horizonte MG, Brazil. E-mail: estevam@dees.ufmg.br

Contract/grant sponsor: CAPES

Contract/grant sponsor: FAPEMIG

Contract/grant sponsor: CNPq
}

CCC 0029-5981/99/341595-13\$17.50

Copyright (C) 1999 John Wiley \& Sons, Ltd.

Received 1 September 1998

Revised 1 February 1999 
of the compounding substances, which implies in performing more tests for the characterization of its material constants when, for example, a new fibre orientation or the inclusion of another phase is considered. The authors adopt the consideration of composite material behaviour as the combined behaviour of the different compounding substances. Each material is considered individually, allowing the plastification of the matrix, for example, independently from the fibres.

Another point to be stressed is that isotropy is an exception rather than a rule when dealing with composites. A simple, general and effective anisotropic model is therefore essential for an efficient large strain materially non-linear finite element algorithm.

In this paper the authors use the mechanical properties of the anisotropic material to define two fourth-order tensors which establish a mapping between the real stress and strain spaces and fictitious, isotropic, stress and strain spaces. As an elastoplastic behaviour is assumed, the yield surface in the fictitious spaces is chosen so as to fulfil the requisites of convexity and invariance, and the simple and well-proven algorithms for numerical integration of isotropic rate constitutive equations can be used. A similar procedure can be used to study materials with damage or creep. The proposed algorithm is implemented in the explicit dynamic code SIMPACT [1], allowing the consideration of contact, treated with the penalty method. As the base programme is explicit, the calculation of stiffness matrices is not necessary. By adding an external loop for the determination of the left-hand side of the dynamic equation, and weighting the contribution of different
substances to the global behaviour, the algorthm followed by the code incorporates the analysis
of composite materials according to the mixing theory [2].
A brief discussion of the mixing theory is given in Section 2, while the basis for the proposed
approach to treat anisotropic materials is given in Section 3. The anisotropic modd yerification
and the main steps for its implenentation are given in Section 4 .

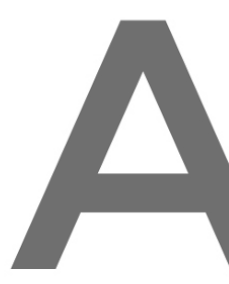

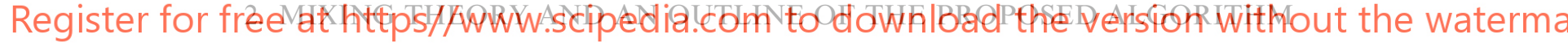

The implemented formulation for large strains considers a multiplicative decomposition of the strain gradient tensor such that

$$
\mathbf{F}=\mathbf{F}^{\mathrm{e}} \mathbf{F}^{\mathrm{p}}
$$

where $\mathbf{F}$ is the strain gradient, $\mathbf{F}^{\mathrm{e}}$ and $\mathbf{F}^{\mathrm{p}}$ its elastic and plastic components. In the original or deformed configuration the usual additive decomposition of strains in its elastic and plastic parts is also assumed, so that, for Almansi strains

$$
\mathbf{e}=\mathbf{e}^{\mathrm{e}}+\mathbf{e}^{\mathrm{p}}
$$

The stress and strain conjugated measures in the deformed configuration are the Kirchoff-Trefftz and Almansi tensors, and the constitutive model assumes an hyperelastic constitutive law

$$
\tau=\rho_{0} \frac{\partial \Psi}{\partial \mathbf{e}^{\mathrm{e}}}
$$

where $\tau$ are the Kirchoff stresses, $\psi\left(\mathbf{e}^{\mathrm{e}}\right)$ the elastic free energy and $\rho_{0}$, the initial mass density.

For anisotropic materials, an assumed one to one invertible transformation is performed to map the real material into an equivalent isotropic material, which is then used in the integration 
of the rate form of constitutive equations. The inverse of the mapping tensor is used to recuperate the anisotropic response.

The other central aspect of the proposed algorithm is the treatment of composite materials using a mixing formulation. The model is described in $[3,4]$ for multiphase materials. The mixing formulation was first proposed by Truesdell and Toupin [5], based on the following assumptions:

(a) Each infinitesimal volume of the composite material is simultaneously occupied by a number $n$ of compounding substances.

(b) The global behaviour of the composite material results from the conjugation of the parallel contribution of each of the substances in proportion to its volume. The resulting model material is then homogeneous.

(c) Strain values at a given point are equal for all substances.

These assumptions allow the uncoupling of the behaviour of the compounding substances so that

$$
\Psi\left(\mathrm{e}^{\mathrm{e}}\right)=\sum_{c=1}^{n} k_{c} \Psi_{c}\left(\mathrm{e}^{\mathrm{e}}\right)
$$

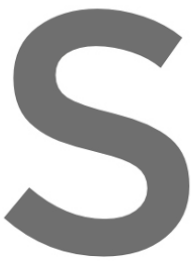

with $k_{\mathrm{c}}$ the relative volum

for each substance, inc

strain elastoplastic ani

substances. An elastic

constitutive model, and th

framework, as described by
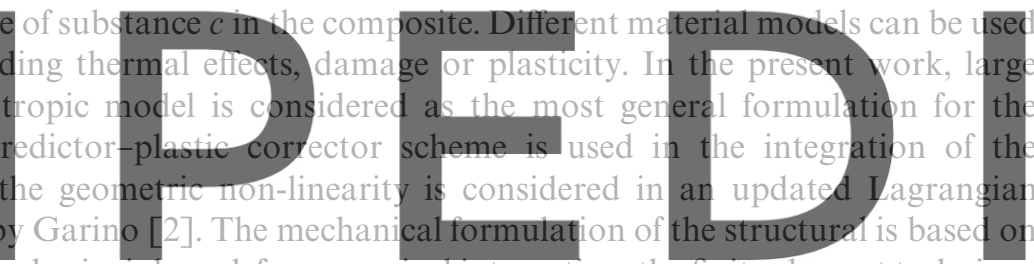

the Hu-Washizu variational principle and, for numerical integration, the finite element technique

is used, including plane and tridimensional solid elements [6] in the framework of an explicit

Register fołyfree atda.ttps//www.scipedia.com to download the version without

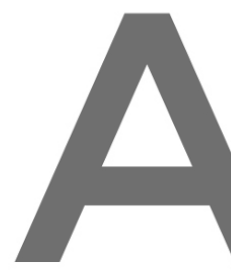

\section{CONCEPT OF MAPPED EQUIVALENT FICTITIOUS ISOTROPIC SPACES}

Anisotropic plasticity models in solid mechanics have usually been developed based on metal plasticity, and often for the specific case of sheet metal forming (see, for example, [7-13]). They involve the definition of a yield surface capable of reproducing the real material behaviour to different degrees of accuracy.

A simplified alternative treatment consists in defining a fictitious isotropic space $\bar{\Omega}$ to which the real, more complex directionally-dependent material behaviour can be mapped. The mapping is performed by fourth-order tensors, as originally proposed by Betten [14]. The mapping tensors should include all the information related to the directional variation of the mechanical properties. Two different mapping tensors are required, one for the stresses

$$
\bar{S}_{I J}=A_{I J K L}^{S} S_{K L}
$$

with $\bar{S}_{i j}$ the mapped second Piola-Kirchoff stress tensor in the fictitious space and $A_{i j k l}^{S}$ the material stress transformation (or mapping) tensor, defined for the undeformed configuration as

$$
A_{I J K L}^{S}=h_{I J}^{\bar{S}}\left(h_{K L}^{S}\right)^{-1}=\bar{S}_{I J}\left(S_{K L}\right)^{-1}
$$


with $h_{I J}^{\bar{S}}$ and $h_{K L}^{S}$, the yield strength tensors in the fictitious and real solids. Tensor $h_{I J}^{\bar{S}}$ can be arbitrarily set by the user, and normally is composed of a set of strengths, which will be considered invariant in the fictitious space and coincident with the real properties. For example the highest values in a given direction real material.

The use of the the mapped stress space to study the material behaviour implies in loosing part of the material data in the analysis, as it is based on a reduced set of parameters. Nevertheless, the simplified approach allows, within its limitations, a flexible tool for the analysis of problems with complex constitutive laws [4].

The second mapping is defined for the strains

$$
\bar{E}_{I J}=A_{I J K L}^{E} E_{K L}
$$

where $\bar{E}_{i j}$ is the Green Lagrange strain tensor in the fictitious space and $A_{i j k l}^{E}$ the material strain transformation tensor. A description of the model for small strains is given in [14]. In the next section the proposed algorithm for large strain analysis is explained.
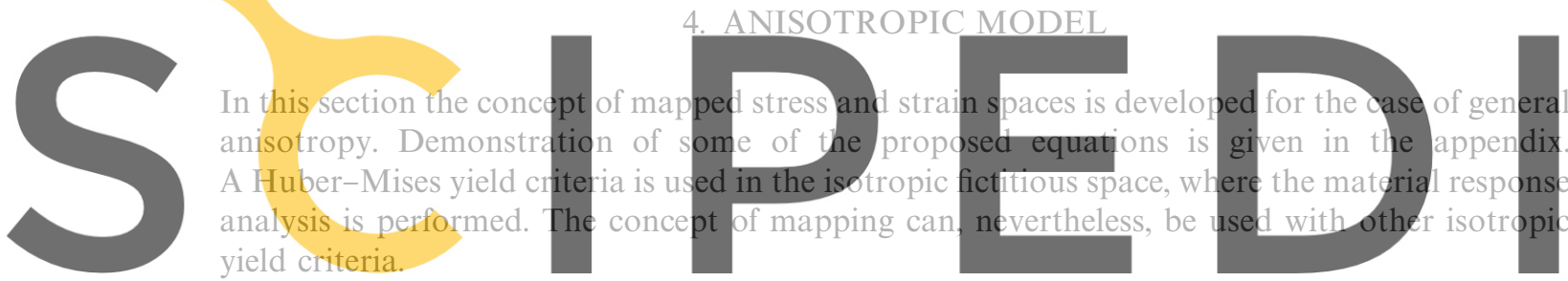
yield criteria.

For a given time increment $\Delta t$, and known displacements ${ }^{t} \mathbf{u}$ and ${ }^{t+\Delta t} \mathbf{u}$, the incremental Register for displacements at are given by

$$
\mathbf{u}={ }^{t+\Delta t} \mathbf{u}-{ }^{t} \mathbf{u}
$$

and the deformed configuration by

$$
{ }^{t+\Delta t} \mathbf{x}={ }^{t} \mathbf{X}+\mathbf{u}
$$

The incremental strain gradient can be found as

$$
{ }^{t+\Delta t} \mathbf{F}^{-1}=\mathbf{I}-\left.\frac{\partial \mathbf{u}}{\partial \mathbf{x}}\right|_{t+\Delta t}
$$

Using the Finger strain tensor ${ }^{t} \mathbf{b}^{\mathbf{e}-1}$, the predicted strains for time $t+\Delta t$ are given by

$$
\left({ }^{t+\Delta t} \mathbf{b}^{\mathrm{e}-1}\right)^{\mathrm{PR}}=\mathbf{F}^{-\mathrm{T} t} \mathbf{b}^{\mathrm{e}-1} \mathbf{F}^{-1}
$$

The predicted elastic Almansi strains and corresponding Kirchoff stresses are then

$$
\begin{aligned}
{ }^{t+\Delta t} \mathbf{e}^{\mathrm{e}} & =\frac{1}{2}\left({ }^{t+\Delta t} \mathbf{g}-{ }^{t+\Delta t} \mathbf{b}^{\mathrm{e}-1}\right) \\
{ }^{t+\Delta t} \tau & =\left.\rho_{0} \frac{\partial \Psi}{\partial \mathbf{e}^{\mathrm{e}}}\right|_{t+\Delta t}
\end{aligned}
$$



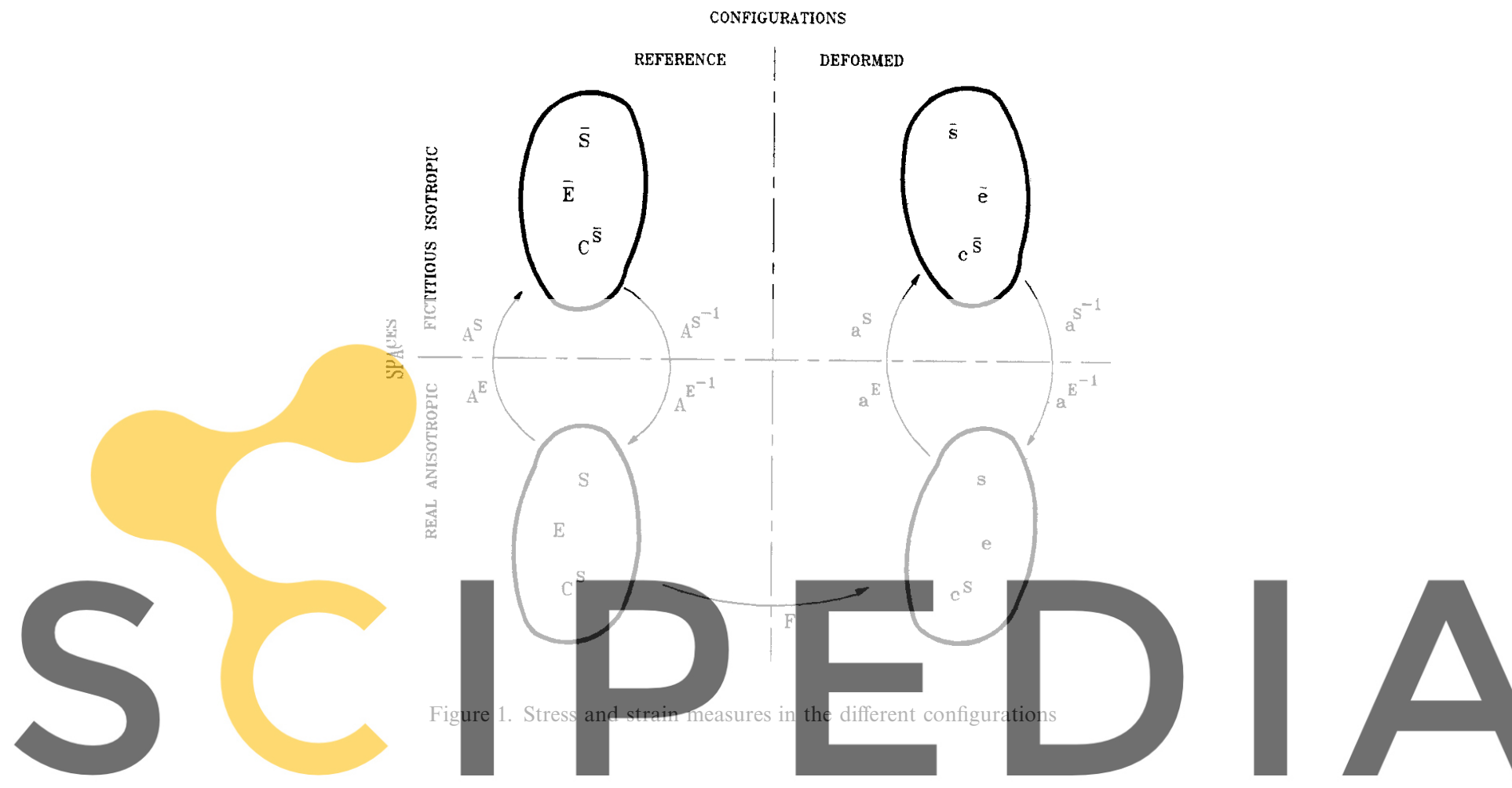

As the Huber-Mises yield function is used in the isotropic fictitious space, only the deviatoric

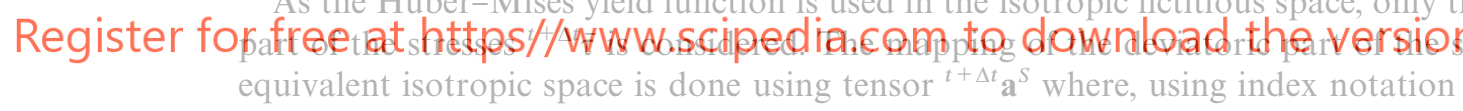

$$
{ }^{t+\Delta t} a_{i j k l}^{S}=\overrightarrow{\vec{\Phi}} A_{I J K L}^{S}=F_{I i} F_{J j} A_{I J K L}^{S} F_{K k}^{-1} F_{L l}^{-1}
$$

with $\overrightarrow{\vec{\phi}}$ the push-forward operation for the fourth-order tensor. Note that $A_{I J K L}^{S}$, defined in the original configuration, does not change with time, while $a_{i j k l}^{S}$, the spatial mapping tensor for stresses, is dependent on time through $F_{I J}$. Figure 1 shows, schematically, in the real and fictitious spaces involved, the four different stresses, their corresponding strain and the mapping tensors between them.

In Figure $1,{ }^{0} \Omega$ is the initial or reference configuration, ${ }^{0} \bar{\Omega}$ the corresponding isotropic configuration, ${ }^{t} \Omega$ the deformed configuration and ${ }^{t} \bar{\Omega}$ the fictitious deformed configuration.

The mapping tensor for strain in the initial configuration, $A_{I J K L}^{E}$, can be obtained as follows:

$$
A_{I J K L}^{E}=\bar{E}_{I J}\left(E_{K L}\right)^{-1}
$$

From equations (7) and (15), and relating the strains $E_{I J}$ to the corresponding stresses $S_{I J}$ through the constitutive fourth-order tensor $C_{I J K L}^{S}\left(C_{I J K L}^{\bar{S}}\right.$ in $\left.\bar{S} \times \bar{E}\right)$ :

$$
\begin{aligned}
A_{I J K L}^{S} & =\left(C_{I J R S}^{\overline{\mathrm{S}}} \bar{E}_{R S}\right)\left(C_{K L M N}^{S} E_{M N}\right)^{-1}=C_{I J R S}^{\bar{S}} A_{R S M N}^{E}\left(C_{K L M N}^{S}\right)^{-1} \\
A_{R S M N}^{E} & =\left(C_{I J R S}^{\bar{S}}\right)^{-1} A_{I J K L}^{S} C_{K L M N}^{S}
\end{aligned}
$$


A push forward is applied on $\mathbf{A}^{E}$, resulting, as shown in the appendix, in

$$
a_{i j l k}^{e}=\overrightarrow{\vec{\Phi}} A_{I J K L}^{E}=F_{I i}^{-1} F_{J j}^{-1} A_{I J K L}^{E} F_{K k} F_{L l}
$$
tion.

The tensor $a_{i j k l}^{e}$ performs the mapping for the fictitious strain space in the deformed configura-

After calculating $a_{i j k l}^{e}$ and $a_{i j k l}^{S}$, the deviatoric part of the Kirchoff stresses in equation (13) is mapped into space $\overline{\mathbf{S}}$. The strain and stress transformations in the spatial configuration described in equations (19) and (20) are derived in the appendix

$$
\begin{aligned}
{ }^{t+\Delta t} \bar{\tau} & ={ }^{t+\Delta t} \mathbf{a}^{S t+\Delta t} \tau \\
{ }^{t+\Delta t} \bar{e} & ={ }^{t+\Delta t} \mathrm{a}^{\mathrm{e} t+\Delta t} e
\end{aligned}
$$

The yield condition is then verified for ${ }^{t+\Delta t} \bar{\tau}$, and if the stresses are outside the defined yield surface $f(\bar{\tau})$, a plastic correction is performed. The integration of the constitutive equation uses the standard radial return algorithm, and the corrected stresses and corresponding Finger strains are mapped back to spaces $\mathbf{S}$ and $\mathbf{E}$. Otherwise, the elastic (and eventually anisotropic) response calculated in equation (14) is assumed and no correction is necessary.

The plastic correction is done on the fictitious space $\overline{\mathrm{S}}$, and the adopted flow rule is non-

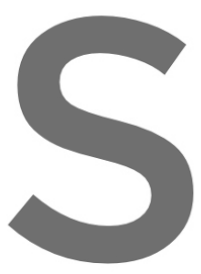
associative. The flow ve
expands in the correct
flow is rotated by the
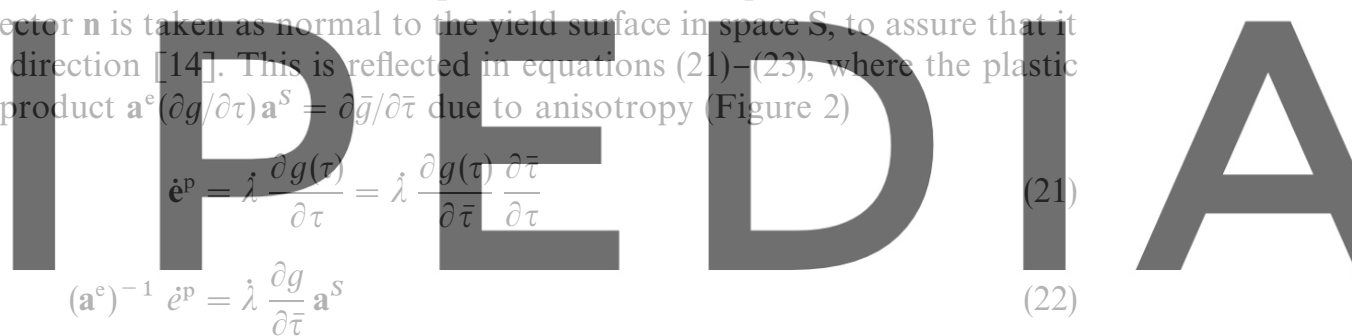

Register for free at https//www.scipedia.com to download the version without the waterma $\dot{\mathrm{e}}^{\mathrm{p}}=\dot{\lambda} \mathrm{a}^{\mathrm{e}} \frac{\partial g(\tau)}{\partial \bar{\tau}} \mathrm{a}^{S}$

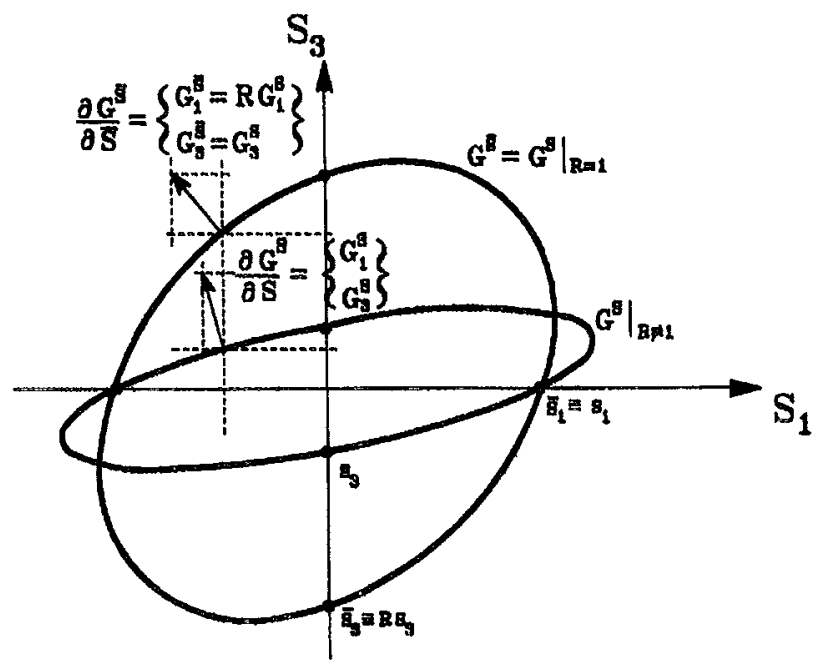

Figure 2. Yield surfaces and flow rule for spaces $\mathbf{S}$ and $\overline{\mathbf{S}}$ 
Then, denoting $\gamma$ as the incremental plastic multiplier, the elastic part of the Finger strain in the fictitious space results from

$$
{ }^{t+\Delta t} \overline{\mathbf{b}}^{\mathrm{e}-1}=\left({ }^{t+\Delta t} \overline{\mathbf{b}}^{\mathrm{e}-1}\right)^{\mathrm{PR}}+2 \gamma^{t+\Delta t} \mathbf{n}
$$

Finally, the corrected stresses $\bar{\tau}$ are found as

$$
{ }^{t+\Delta t} \bar{\tau}=\left.\rho \frac{\partial \bar{\psi}\left(\overline{\mathbf{e}}^{\mathrm{e}}\right)}{\partial \overline{\mathbf{e}}^{\mathrm{e}}}\right|_{t+\Delta t}
$$

Different choices of the free energy function $\bar{\psi}$ can be made. In the present version of the program, a von Mises yield surface is used, with the option to include linear or non-linear isotropic hardening.

The new corrected stress and strain tensors are mapped back to the spaces $\mathbf{s}$ and $\mathbf{e}$ using the inverse transformations

$$
\begin{aligned}
{ }^{t+\Delta t} \tau & =\left({ }^{t+\Delta t} \mathbf{a}^{s}\right)^{-1}{ }^{t+\Delta t} \bar{\tau} \\
{ }^{t+\Delta t} \mathbf{b}^{\mathrm{e}-1} & =\left({ }^{t+\Delta t} \mathbf{a}^{e}\right)^{-1 t+\Delta t} \overline{\mathbf{b}}^{\mathrm{e}-1}
\end{aligned}
$$

When using the mixing theory for the analysis of composites, at this stage new iterations are

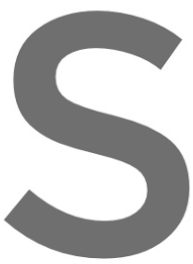
performed for the analy
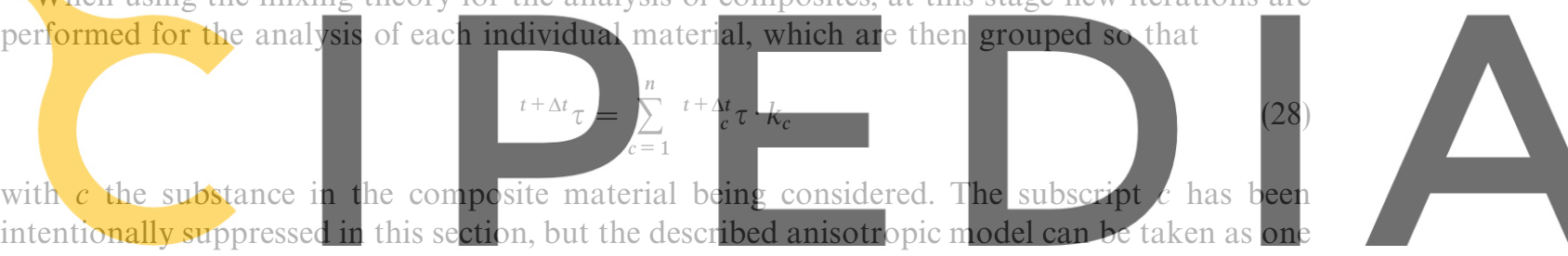

(or more) of the substances in a particular composite material.

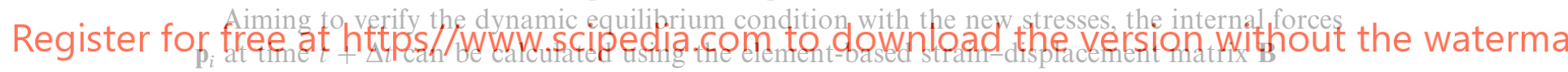

$$
{ }^{t+\Delta t} \mathbf{p}_{i}=\int_{{ }^{\prime} \Omega} B^{\mathrm{T}}{ }^{t+\Delta t} \tau \mathrm{d} \Omega
$$

\section{EXAMPLE PROBLEMS}

Three examples of applications are shown in this item. The first is a two-phase material with an isotropic matrix and long fibres; the second a laminated plate submitted to an impulsive load. The third example shows an application of the mapped anisotropy.

\subsection{Two-phase bar}

In this example, a bar, modeled with 40 brick eight-node elements is submitted to an axial displacement. The two materials are described below

Matrix: $E_{x}=E_{y}=E_{z}=7 \cdot 24 \mathrm{tf} / \mathrm{mm}^{2}$

$$
\begin{aligned}
& f_{x}=0.036 \mathrm{tf} / \mathrm{mm}^{2} \\
& v=0.33, k_{c}=80 \%
\end{aligned}
$$




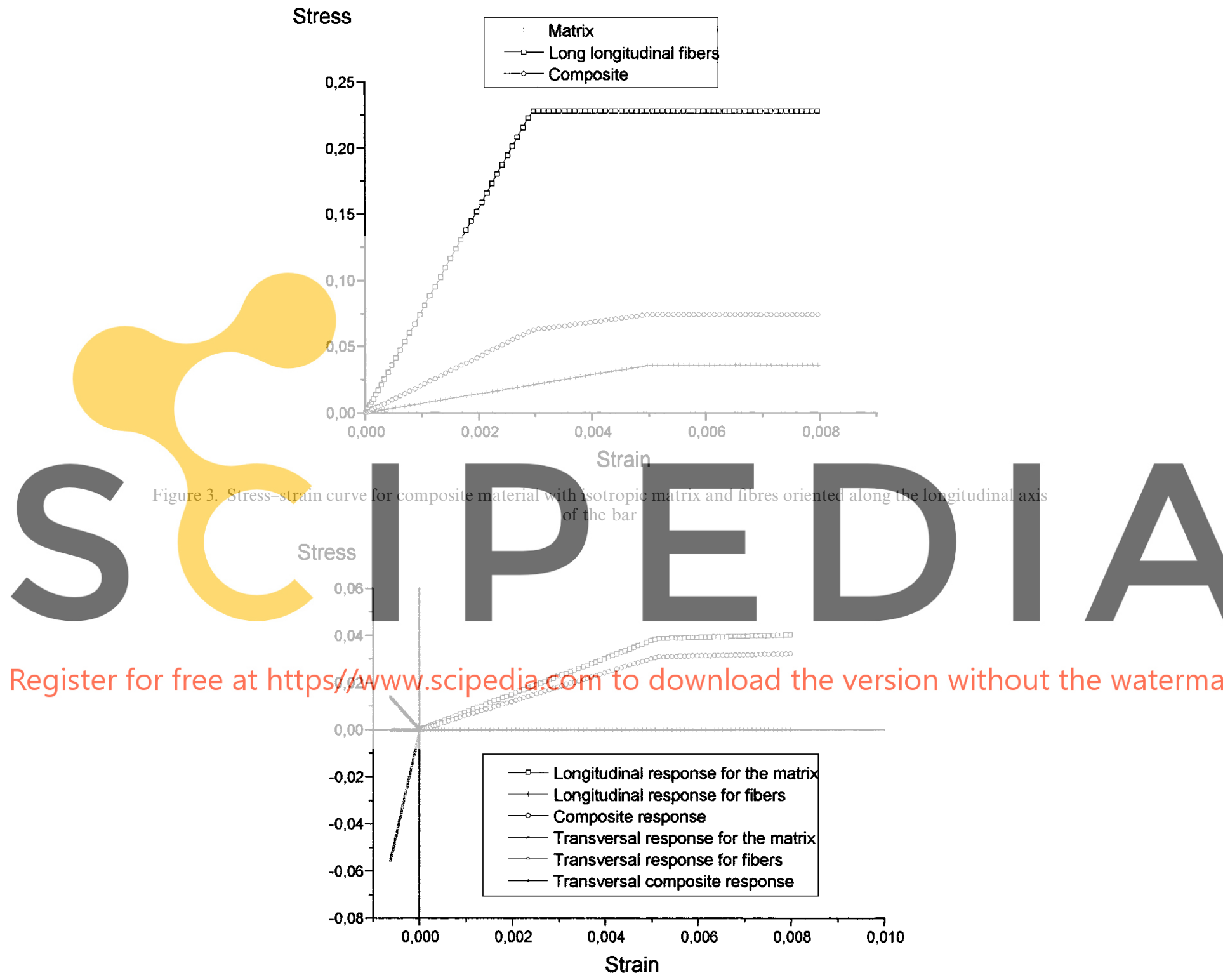

Figure 4. Stress-strain curve for isotropic matrix and fibres oriented along the axis. Negative stress values correspond to transversal direction

Material 2: $E_{x}=84.4 \mathrm{tf} / \mathrm{mm}^{2}$

$$
\begin{gathered}
f_{x}=0 \cdot 2283 \mathrm{tf} / \mathrm{mm}^{2} \\
v=0 \cdot 30, k_{c}=20 \%
\end{gathered}
$$

The resulting response is shown in Figures 3 and 4. In the latter, the fibres are oriented transversally to the direction of the extension, therefore reducing the amount of resisting material 


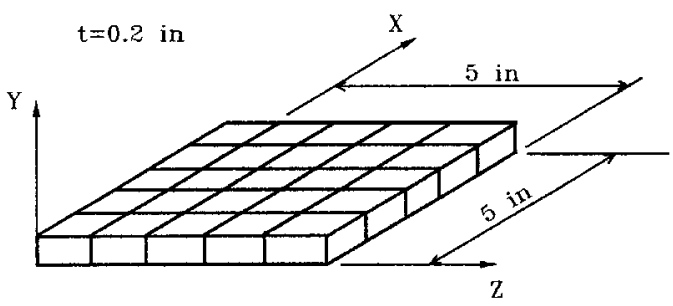

Figure 5. Model for impact problem with $1 / 4$ of simply supported composite plate with hexahedral solid element discretization
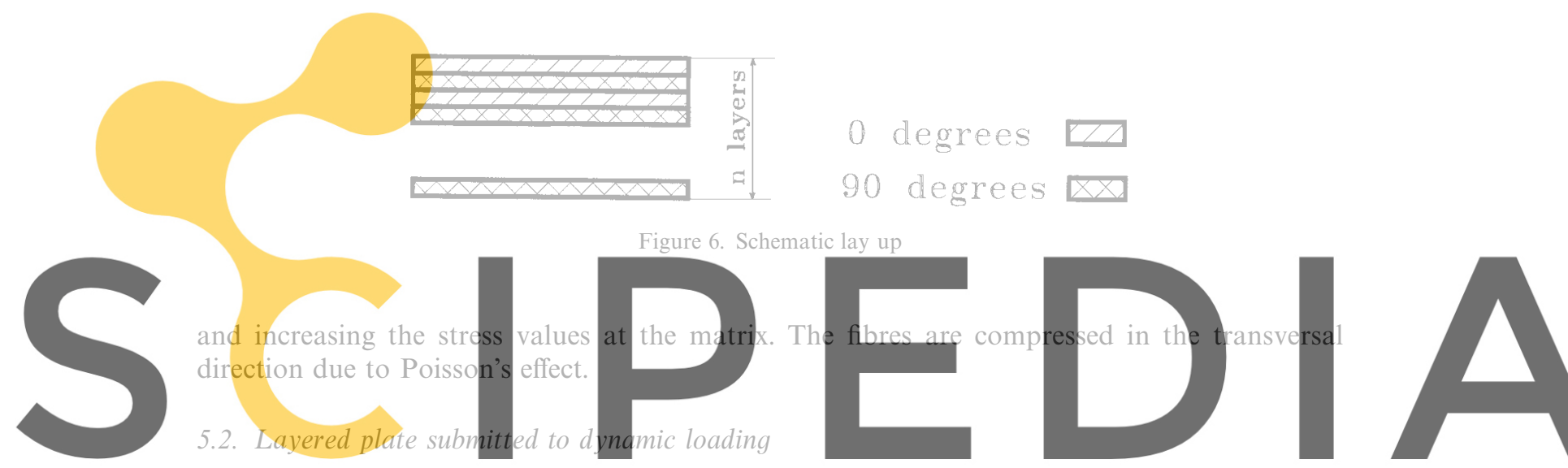

In the second example, the plate shown in Figure 5 is submitted to an impulsive distributed

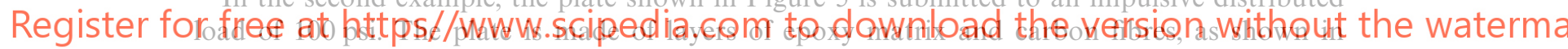
Figure 6.

For comparison, an equivalent isotropic homogenized material is considered, obtained from the volume participation of the fibre and matrix. The considered materials are

Epoxy resin: $E=1 \times 10^{6} \mathrm{psi}, \sigma_{y}=5.8 \times 10^{3} \mathrm{psi}, v=0 \cdot 4, \rho=0.04 \mathrm{lb} / \mathrm{in}^{3}$

Carbon fibre: $E=4 \times 10^{7}$ psi, $\sigma_{y}=3.04 \times 10^{4}$ psi, $v=0 \cdot 2, \rho=0.082 \mathrm{lb} / \mathrm{in}^{3}$

Homogenized material: $E=2.05 \times 10^{7} \mathrm{psi}, \sigma_{y}=1 \cdot 81 \times 10^{4} \mathrm{psi}, v=0 \cdot 3, \rho=0.061 \mathrm{lb} / \mathrm{in}^{3}$

Figure 7 shows the obtained response for the centre point of the composite using the described model. In Figure 8, the displacement versus time graphic of the plate is given. Notice that the capability of detecting yielding in each of the materials provides a deviation from the consideration of a single, equivalent material model. The fact that yield limits are considerably different causes the non-linear behaviour to be detected at the time the matrix reaches its yield stress, while the fibres still behave elastically.

\subsection{Spherical cap under impulsive loading}

In this example, an anisotropic spherical cap, shown in Figure 9, is submitted to an impulsive distributed load of 600 psi. Forty-eight four-noded axisymmetric elements were used in the 


\section{Center displacements (in.)}
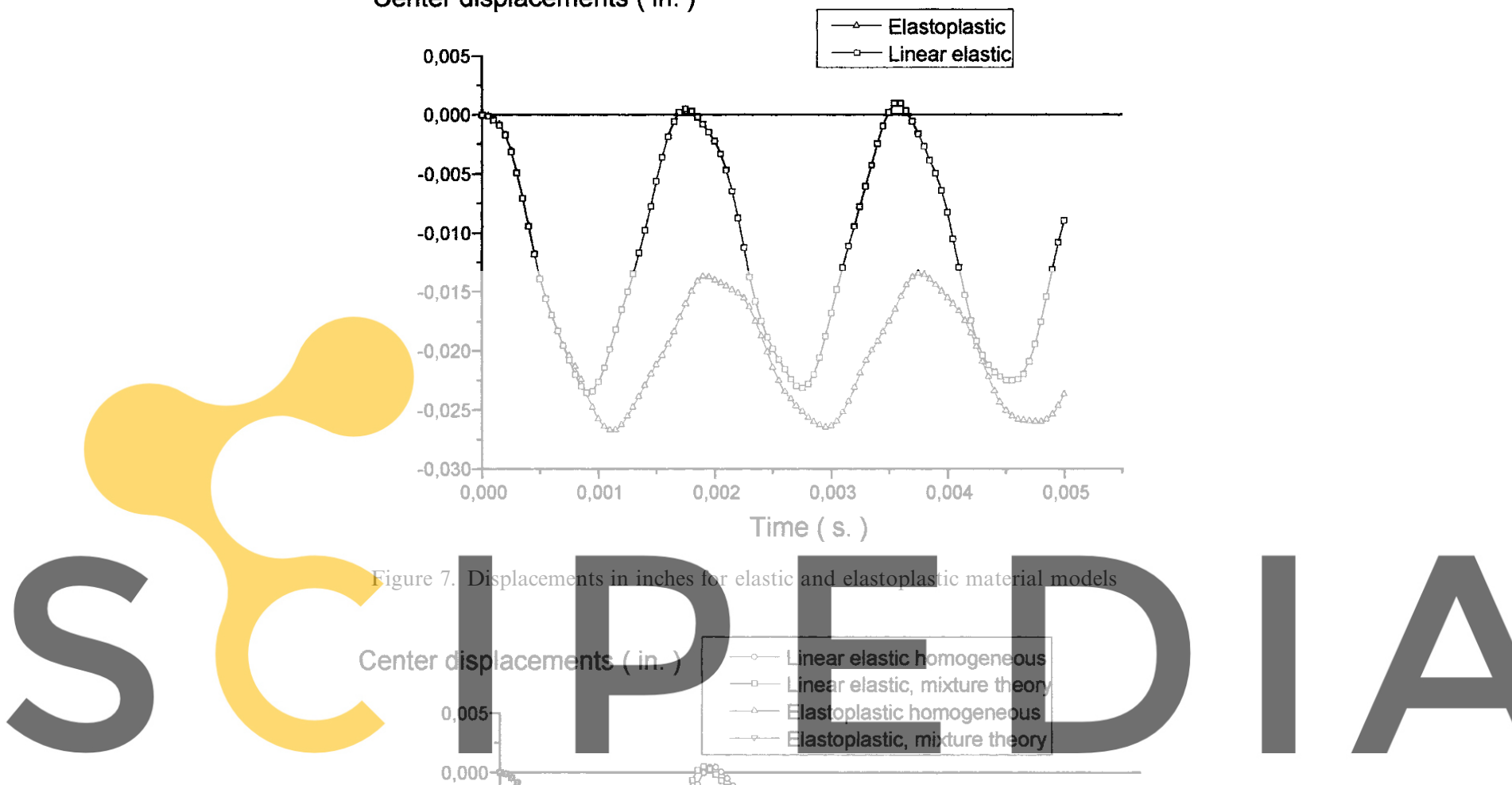

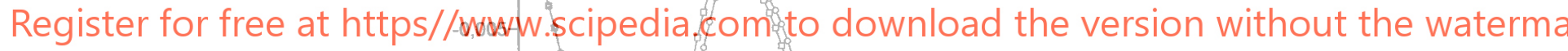

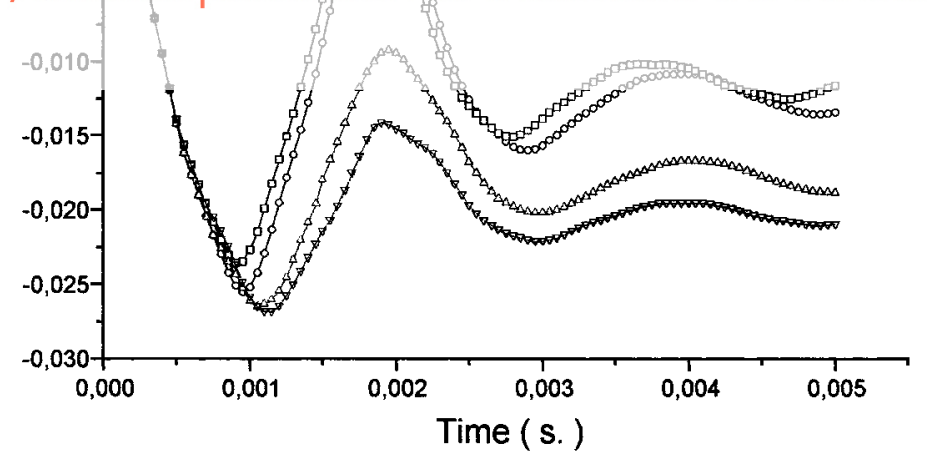

Figure 8. Deflections in inches of the plate with different material models

analysis, which is performed with and without updating the mapping tensor to account for large strains.

The considered material data is:

$$
\begin{aligned}
E & =10 \cdot 5 \times 10^{6} \mathrm{psi}, H=0.21 \times 10^{6} \mathrm{psi}, \rho=2.45 \times 10^{-4} \mathrm{lb} \mathrm{s}^{2} / \mathrm{in}^{2}, v=0.30 \\
\sigma_{00} & =24 \times 10^{4} \mathrm{psi}, \sigma_{90}=4 \times 10^{4} \mathrm{psi}, \sigma_{45}=24 \times 10^{4} \mathrm{psi}
\end{aligned}
$$




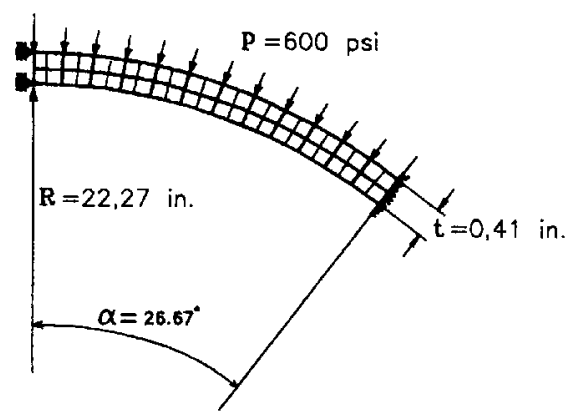

Figure 9. Spherical cap

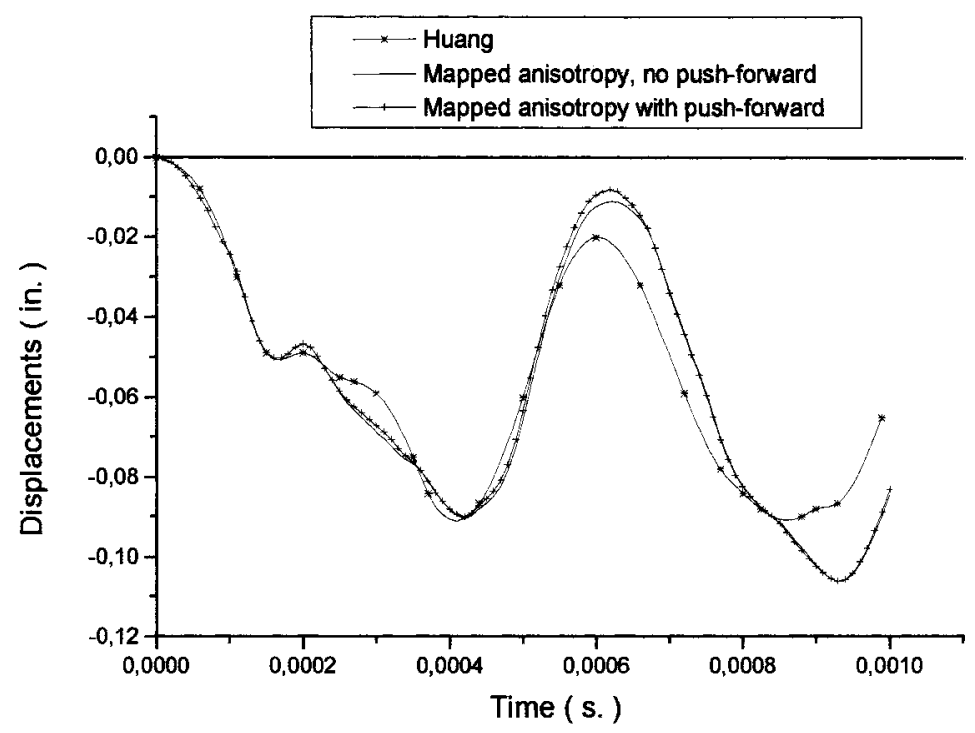

Figure 10. Results from [15], and present with and without updating of mapping tensors

The results indicate a good agreement with these described by Huang [15], who used Hill's criterion for yielding. The effect of considering the push forward of the mapping tensors does not affect significantly the response, as expected due to the small physical non-linearity.

\section{CONCLUSIONS}

The examples indicate that the proposed analysis procedure is valid and quite general alternative for composite structures, as well as for anisotropy. Further tests and comparison with experimental results should be performed to access its range of applicability and limitations. 


\section{APPENDIX}

Some of the expressions in item 4 are demonstrated in this appendix. The first equation (14), detailing the push-forward operation of tensor $A_{I J K L}^{E}$, is derived. The pull-back operation is given by

$$
\begin{aligned}
& \bar{E}_{I J}=F_{I i} F_{J j} \bar{e}_{i j} \\
& E_{I J}=F_{I i} F_{J j} e_{i j}
\end{aligned}
$$

Inverting (33)

$$
E_{J I}^{-1}=e_{i j}^{-1} F_{J j}^{-1} F_{I i}^{-1}
$$

Substituting the expressions in (32) and (33) in (15)

$$
A_{I J K L}^{E}=F_{I i} F_{J j} \bar{e}_{i j} e_{k l}^{-1} F_{L l}^{-1} F_{K k}^{-1}
$$

Taking $a_{i j k l}^{e}=\bar{e}_{i j} e_{k l}^{-1}$

$$
A_{I J K L}^{E}=F_{I i} F_{J j} a_{i j k l}^{E} F_{L l}^{-1} F_{K k}^{-1}
$$

The push-forward of $A_{I J K L}^{E}$ is then given by

$$
{ }^{t+\Delta t} a_{i j k l}^{e}=\overrightarrow{\widetilde{\Phi}} A_{I J K L}^{E}=F_{I i}^{-1} F_{J j}^{-1} A_{I J K L}^{E} F_{K k} F_{L l}
$$

Next, the expression in (14) for the push-forward of the mapping tensor $A_{I J K L}^{S}$ is obtained. The push-forward of the contravariant second-order Kirchoff stress tensor is derived as follows:

$$
\begin{aligned}
\bar{\tau}_{i j} & =F_{I i} F_{J j} \bar{S}_{I J} \\
\bar{S}_{I J} & =F_{J j}^{-1} F_{I i}^{-1} \bar{\tau}_{i j} \\
\bar{S}_{L K}^{-1} & =\bar{\tau}_{l k}^{-1} F_{K k} F_{L l}
\end{aligned}
$$

From the definition in (5), and using (39) and (40)

$$
\begin{aligned}
A_{I J K L}^{S} & =F_{J j}^{-1} F_{I i}^{-1} \bar{\tau}_{i j} \tau_{l k}^{-1} F_{K k} F_{L l}=F_{J j}^{-1} F_{I i}^{-1} a_{i j l k}^{S} F_{K k} F_{L l} \\
a_{i j k l}^{S} & =F_{I i} F_{J j} A_{I J K L}^{S} F_{K k}^{-1} F_{L l}^{-1}
\end{aligned}
$$

Another point that can be verified is the equivalence between equations (17) and (41).

The constitutive tensors in the initial and deformed configurations are related by

$$
\begin{aligned}
c_{i j k l}^{S} & =F_{i I} F_{j J} F_{k K} F_{l L} C_{I J K L}^{S} \\
C_{I J K L}^{\bar{S}} & =F_{L l}^{-1} F_{K k}^{-1} F_{J j}^{-1} F_{I i}^{-1} c_{i j k l}^{\bar{S}} \\
\left(C_{K L M N}^{S}\right)^{-1} & =\left(c_{k l m n}^{S}\right)^{-1} F_{k K} F_{l L} F_{m M} F_{n N}
\end{aligned}
$$

Substituting equations (44), (45) and (36) into (16)

$$
A_{I J K L}^{S}=F_{J j}^{-1} F_{I i}^{-1} c_{i j r s}^{\bar{S}} a_{r s m n}^{E}\left(c_{k l m n}^{S}\right)^{-1} F_{k K} F_{l L}=F_{J j}^{-1} F_{I i}^{-1} a_{i j k l}^{S} F_{k K} F_{l L}
$$

where $a_{i j k l}^{S}=c_{i j r s}^{\bar{S}} a_{r s m n}^{E}\left(c_{k l m n}^{S}\right)^{-1}$. 
Equation (46) corresponds to the previously obtained expression in equation (42) for $a_{I J K L}^{S}$. The mapping of Kirchoff stresses expressed by equation (19) is shown in the next lines. Starting from (5), using equation (39) on both sides of the equation, we obtain

$$
\begin{aligned}
F_{J j}^{-1} F_{I i}^{-1} \bar{\tau}_{i j} & =A_{I J K L}^{S} F_{L l}^{-1} F_{K k}^{-1} \tau_{k l} \\
\bar{\tau}_{i j} & =F_{I i} F_{J j} A_{I J K L}^{S} F_{L l}^{-1} F_{K k}^{-1} \tau_{k l}
\end{aligned}
$$

Using (14)

$$
\bar{\tau}_{i j}=a_{i j k l}^{S} \tau_{k l}
$$

Similarly, equation (20) can be demonstrated.

\section{ACKNOWLEDGEMENTS}

Estevam B. Las Casas and José B. Rubert would like to acknowledge the funding provided by the Brazilian agencies CAPES, FAPEMIG and CNPq for the development of this work.

\section{REFERENCES}

1. Miquel J, Oñate E, Garcia Garino C, Botello S, Flores F, Rojek J. Analysis of shock and Impact Problems between Deformable Solids using the Finite Element Method-project SIMPACT CIMNE report 25, 1994 (in Spanish).

2. Garcia Garino C, Oliver J. A numerical model for elastoplastic large strain problems-fundamentals and applications. In Computational Plasticity, Owen R, Oñate E, Hinton E (eds). Vol. 1. Pineridge Press-CIMNE, 1992; 117-129.

3. Oller S, Oñate E, Miquel J. A mixing anisotropic formulation for analysis of composites. Communications in Numerical Methods in Engineering 1996; 12(8):471-482.

4. Rubert JB. Large strain and anisotropy by mapping tensors applied to composites and metal forming. Ph.D. Dissertation, EESC-USP, Universidade de São Paulo, São Carlos, Brazil (in Portuguese).

5. Trusdell C, Toupin R. The classical field theories. In Handbuch der Physik III/I, Flügge S (ed.). Springer: Berlin, 1960.

6. Garcia Garino C. A Numeric Model for the Analysis of Elastoplastic Solids under Large Displacements. Ph.D. Dissertation, School of Civil Engineering, Universitat Politècnica de Catalunya, 1993 (in Spanish).

7. Hill R. A theory of the yielding and plastic flow of anisotropic metals. Proceedings of the Royal Society of London Series A 1948; 193:281-297.

8. Barlat F, Lian J. Plastic behaviour and strechability of sheet metals. Part I-A yield function for orthotropic sheets under plane stress conditions. International Journal of Plasticity 1989; 5:51-66.

9. Chung K, Shah K. Finite element simulation of sheet metal forming for planar anisotropic metals. International Journal of Plasticity 1992; 8:453-476.

10. Barlat F, Chung K, Shah KN, Lege DJ, Brem JC. Modelling of sheet forming of polycrystaline metals. 2nd World Congress on Computer Mechanics, Stuttgart, Germany, 1990 pp. 383-386. Hill R. Theoretical plasticity of textured aggregates. Mathematical Proceedings of the Cambridge Philosophical Society 1979; 85:179-191.

11. Chou CH, Pan J, Tang SC. An anisotropic stress resultant constitutive law for sheet metal forming. International Journal for Numerical Methods in Engineering 1996; 39:435-449.

12. Hill R. Constitutive modelling of orthotropic plasticity in sheet metals. Journal of Mechanics, Physics and Solids 1990; 38(3):405-417.

13. Betten J. Creep theory of anisotropic solids. Journal of Rheology 1981; 25(6):565-581.

14. Oller S, Botello S, Miquel J, Oñate E. An anisotropic elastoplastic model based on an isotropic formulation. Engineering Computations 1995; 12:245-262.

15. Huang HC. Static and Dynamic Analysis of Plates and Shells. Springer: Berlin, 1989. 